\title{
On the extension of the Painlevé property to difference equations
}

\author{
M J Ablowitz $\dagger$, R Halburd $\dagger$ † and B Herbst $\$$ \\ $\dagger$ Department of Applied Mathematics, University of Colorado, Boulder, CO 80309-526, USA \\ \$ Department of Mathematical Sciences, Loughborough University, Loughborough, \\ Leicestershire LE11 3TU, UK \\ $\S$ Department of Applied Mathematics, University of Stellenbosch, Stellenbosch 7602, \\ South Africa
}

Received 25 May 1999, in final form 11 January 2000

Recommended by Y Kuramoto

\begin{abstract}
It is well known that the integrability (solvability) of a differential equation is related to the singularity structure of its solutions in the complex domain - an observation that lies behind the Painlevé test. A number of ways of extending this philosophy to discrete equations are explored. First, following the classical work of Julia, Birkhoff and others, a natural interpretation of these equations in the complex domain as difference or delay equations is described and it is noted that arbitrary periodic functions play an analogous role for difference equations to that played by arbitrary constants in the solution of differential equations. These periodic functions can produce spurious branching in solutions and are factored out of the analysis which concentrates on branching from other sources. Second, examples and theorems from the theory of difference equations are presented which show that, modulo these periodic functions, solutions of a large class of difference equations are meromorphic, regardless of their integrability. It is argued that the integrability of many difference equations is related to the structure of their solutions at infinity in the complex plane and that Nevanlinna theory provides many of the concepts necessary to detect integrability in a large class of equations. A perturbative method is then constructed and used to develop series in $z$ and the derivative of $\log \Gamma(z)$, where $z$ is the independent variable of the difference equation. This method provides an analogue of the series developed in the Painlevé test for differential equations. Finally, the implications of these observations are discussed for two tests which have been studied in the literature regarding the integrability of discrete equations.
\end{abstract}

AMS classification scheme numbers: 30D35, 39A10, 39A12

\section{Introduction}

An ordinary differential equation (ODE) is said to possess the Painlevé property if all of its solutions are single-valued about all movable singularities. A singularity is said to be movable if it varies with initial conditions, i.e. it is not a singularity of the equation itself. The observation that this property is related to integrability has proved most fruitful, cf [4-7,27]. It is widely believed that all ODEs that possess the Painlevé property are integrable (i.e. solvable) either explicitly or via a related linear problem. We also remark, however, that certain equations solvable via an evolving monodromy problem do not possess the Painlevé property $[1-3,10]$.

Painlevé and his colleagues identified all second-order ODEs within a large class that possess the Painlevé property $[16,17,30,31]$. All of these equations were integrated in terms 
of known functions except six which are now known as the Painlevé equations $\left(P_{\mathrm{I}}-P_{\mathrm{VI}}\right)$. The first two of these are

$$
\begin{array}{ll}
P_{\mathrm{I}} & \frac{\mathrm{d}^{2} y}{\mathrm{~d} z^{2}}=6 y^{2}+z \\
P_{\mathrm{II}} & \frac{\mathrm{d}^{2} y}{\mathrm{~d} z^{2}}=2 y^{3}+z y+\alpha
\end{array}
$$

where $\alpha$ is a constant.

The six Painlevé equations are integrable (solvable) via associated (linear) isomonodromy problems $[14,23]$. There are many ways in which one could discretize these equations (Euler's method, for example). Most of these will no longer possess the many special properties that the differential equations (1) and (2) possess; e.g. related isomonodromy problems and solutions that are asymptotically well behaved. However, a number of discretizations of equations (1) and (2) have been studied in the literature and are believed to be integrable (i.e. solvable via an associated linear problem) $[15,24,26,29,32,33]$. For example,

$$
\begin{aligned}
& y_{n+1}+y_{n}+y_{n-1}=\frac{\alpha n+\beta+\gamma(-1)^{n}}{y_{n}}+\mu \\
& \frac{\alpha(n+1)+\beta}{y_{n+1}+y_{n}}+\frac{\alpha n+\beta}{y_{n}+y_{n-1}}=-y_{n}^{2}+\gamma \\
& y_{n+1}+y_{n-1}=\frac{\alpha n+\beta}{y_{n}}+\frac{\gamma}{y_{n}^{2}} \\
& y_{n+1}+y_{n-1}=\frac{\alpha n+\beta}{y_{n}}+\gamma
\end{aligned}
$$

where $\alpha, \beta$ and $\gamma$ are constants, are some known well behaved discretizations of $P_{\mathrm{I}}$ and

$$
\begin{aligned}
& y_{n+1}+y_{n-1}=\frac{(\alpha n+\beta) y_{n}+\gamma}{1-y_{n}^{2}} \\
& \frac{\alpha n+\beta}{y_{n} y_{n+1}+1}+\frac{\alpha(n-1)+\beta}{y_{n-1} y_{n}+1}=\frac{1}{y_{n}}-y_{n}+\alpha n+\gamma
\end{aligned}
$$

are some known special discretizations of $P_{\mathrm{II}}$.

A natural question arises: is it possible to determine a priori whether a given difference equation is integrable? In the case of differential equations, at least a partial answer is provided by the Painlevé test cf [4-6,27]. This property is suggested by the inverse scattering transform, however, it is still not fully understood why the integrability of a real differential equation should be reflected in the singularity structure of its solutions in the complex domain.

The purpose of this paper is to analyse the behaviour of solutions of difference equations in the complex domain through a number of examples and by exploring the implications of several known theorems. This problem was studied by Nörlund, Birkhoff, Batchelder and others earlier this century for linear difference equations. Julia, Nörlund, and more recently, Kimura [25], Shimomura [37] and Yanagihara [40,41], have derived a number of valuable results for nonlinear difference equations.

We undertake this investigation with the intention of identifying a structure or structures that can be associated with the integrability (or non-integrability) of the difference equation in the spirit of the Painlevé property for ODEs. That is, we wish to find conditions, framed in the language of complex analysis, under which a difference equation would be considered to be of Painlevé type. Some of the conclusions to which we are led are the following. 
(a) Arbitrary periodic functions play an analogous role in the solutions of difference equations to that played by constants in the solutions of ODEs and arbitrary functions on characteristic functions on characteristic manifolds in solutions of PDEs. This classical observation plays a central role in our analysis.

(b) Apart from branching due to the above-mentioned arbitrary periodic functions, the solutions of many difference equations can be extended to the complex plane and are meromorphic.

(c) The integrability of a large class of difference equations is associated with the asymptotic structure of its solutions at infinity.

(d) Nevanlinna theory provides a number of important concepts and tools that can be used as detectors of equations that are integrable. In particular, the order of solutions plays a central role.

(e) Perturbative techniques which are difference versions of those used by Painlevé can be constructed and used to find expansions of solutions that are analogues of the Laurent/psiseries for differential equations.

The tests we describe are not necessary conditions for integrability, just as the Painlevé property is not necessary for the integrability of a differential equation.

Recently, two tests have been proposed as discrete analogues of the Painlevé test. The singularity confinement method of Grammaticos and co-workers $[18,36]$ involves examining solutions (sequences of iterates) of a discrete equation which pass arbitrarily close to a singular point of the equation. The associated test is applicable to a certain class of equations (reversible, strictly rational) and associates integrability with the preservation of information beyond the singularity. This test has been used successfully to find a number of integrable discrete equations. Later we discuss how our analytical observations can be related to this singularity confinement method. Also, the discrete Painlevé property has been introduced by Conte and Musette together with an associated test $[11,12]$. We will explore some of the strengths and weaknesses of this property and how it relates to our asymptotic analysis.

This paper is structured as follows. In section 2 we discuss why difference (or delay) equations allow a natural extension of their solutions to the complex domain as opposed to discrete (or lattice) equations which are defined on the integers only. Thus we adopt the approach of Birkhoff $e t$ al. In this setting arbitrary functions of period $h / m$ (where $h$ is the step size and $m$ is some positive integer) play a role analogous to that played by constants in the theory of differential equations. These periodic functions can introduce complicated branching into solutions of the most benign difference equations and so they must be 'factored out' or otherwise dealt with in the analysis as we are only concerned with singularities other than those generated by the periodic functions. In some sense, branching due to the arbitrary periodic functions in solutions of difference equations is analogous to branching about characteristic manifolds in solutions of partial differential equations.

After factoring out possible branching due to the periodic functions we note that the solutions of a large class of difference equations (including many chaotic examples) are meromorphic in the finite plane. This leads us to the conclusion that for a great many difference equations the natural place where the integrability (or otherwise) could be encoded is in the structure of the singularity at infinity. This suggests that Nevanlinna theory (value distribution theory for meromorphic functions) is an important ingredient in the study of integrable discrete or difference systems.

In section 3 we describe some fundamental concepts from Nevanlinna theory and we note that all meromorphic solutions of many classes of integrable differential and difference 
equations, including the important examples of the Riccati and Painlevé equations, are of finite order. Section 4 describes, via examples, a method to obtain a series representation of a solution of a difference equation that is the natural analogue of the expansions obtained in Painlevé's analysis of differential equations. In section 5 we discuss some of the existing tests that appear in the literature in light of our results.

\section{Difference equations in the complex domain}

We begin by examining some simple discrete equations and the problems that arise when we try to extend their solutions to the complex domain. Consider the discrete (or lattice) equation

$$
u_{n+1}-u_{n}=\frac{1}{(n-c)^{N}} .
$$

If we specify $u_{0}$ we can determine $u_{n}$ for all $n \in \mathbb{N}$, namely

$$
u_{n}=u_{0}+\sum_{i=0}^{n-1} \frac{1}{(i-c)^{N}} .
$$

Note that this solution involves an unbounded number of terms and, from a calculational point of view, involves as much work to evaluate as iterating equation (3). Note that if $c$ is a positive integer then $u_{n}$ is infinite for all $n \geqslant c$. This is also related to the singularity confinement property which we discuss in section 5.1.

We wish to explore the singularity structure of solutions to equations such as (3) in the complex domain. There appears to be no compelling way to extend the solution (4) off the integers uniquely because the independent variable, $n$, appears as an endpoint in the sum. Since equation (3) is only defined on the integers, there is no constraint on extensions $f(z)$ of $u_{n}$ to the complex domain $\left(f(n)=u_{n}, n \in \mathbb{N}\right)$.

We find it convenient to reinterpret discrete (or lattice) equations such as (3) as difference (or delay) equations such as

$$
y(z+1)-y(z)=\frac{1}{(z-c)^{N}}
$$

where $z$ is complex. The homogeneous part of equation (5) is

$$
\Delta y(z):=y(z+1)-y(z)=0 .
$$

The general solution of this equation is any function of period 1 (or $1 / m$ for some positive integer $m$ ). In fact, it is well known that arbitrary periodic functions (which we refer to later as $\pi(z))$ play the same role in solutions of difference equations as that played by arbitrary constants in the solutions of differential equations. Since these functions can introduce any type of singularity into solutions of trivial difference equations we will factor them out of our analysis.

Recall that the gamma function is meromorphic and satisfies

$$
\Gamma(z+1)=z \Gamma(z) .
$$

On taking the logarithm of equation (6) we obtain

$$
\log \Gamma(z+1)-\log \Gamma(z)=\log z
$$


Replacing $z$ with $z-c$ in equation (7) and differentiating $N$ times with respect to $z$, we see that the general solution of equation (5) is

$$
y(z)=\frac{(-1)^{N-1}}{(N-1) !}\left(\frac{\mathrm{d}}{\mathrm{d} z}\right)^{N} \log \Gamma(z-c)+\pi(z)
$$

where $\pi$ is a periodic function (as described above). It is important to note that other than the singularities due to $\pi$, the only singularities of $y(z)$ are poles. This contrasts markedly with the solution of the analogous differential equation

$$
\frac{\mathrm{d} y}{\mathrm{~d} z}=\frac{1}{(z-c)^{N}}
$$

which, in the case $N=1$, has a logarithmic singularity at $z=c$.

Since essential branching (i.e. branching that does not arise from the arbitrary periodic function) is absent in the solutions of the first-order linear difference equation (9), in stark contrast to the differential case, we are naturally led to ask whether essential branching is absent from nonlinear difference equations. Note that equations (5) and (9) have fixed singularities at $z=c$. Throughout the rest of this paper we consider only equations whose solutions have movable singularities. Although there is to date no complete theory of difference equations, the following results are of importance. These theorems were largely motivated by the work of Kimura [25] on the iteration of analytic functions.

Theorem 1 (Shimomura [37]). For any polynomial $P(y)$, the difference equation

$$
y(z+1)=P(y(z))
$$

has a non-trivial entire solution.

Theorem 2 (See Yanagihara [40]). For any rational function $R(y)$, the difference equation

$$
y(z+1)=R(y(z))
$$

has a non-trivial meromorphic solution.

Yanagihara has extended theorem 2 to a limited class of higher-order difference equations [41]. Note that since equations (10) and (11) are autonomous then the general solution of equation (10) is a particular entire function of $z-\pi(z)$ and the general solution of equation (11) is a particular meromorphic function of $z-\pi(z)$, where $\pi(z)$ is an arbitrary function of period one.

Theorem 1 says that even the quintessential chaotic difference equation, the logistic equation $[8,28]$,

$$
y(z+1)=\mu y(z)(1-y(z))
$$

where $\mu$ is a parameter, which includes the (non-integrable) discretization of the Riccati equation (when $\mu=1$ ),

$$
y(z+1)-y(z)+y^{2}(z)=0
$$

has solutions of the form

$$
y(z)=w_{\mu}(z-\pi(z))
$$

where $w_{\mu}$ is a non-trivial entire function (which depends on the choice of parameter $\mu$ ). Viewed as a map, equation (12) is chaotic on its (non-empty) Julia set (see, for example, [13]). If all 
the solutions of equation (12) have this form then the only singularities of $y$ arise from the periodic function $\pi$.

Thus we see that the same (meromorphic) singularity structure in the finite complex plane is found throughout a wide class of difference equations which includes both integrable and non-integrable examples. The solutions of these equations show no essential branching (i.e. branching due to sources other than the arbitrary periodic functions). It appears that for this class the only way in which solutions can differ in their singularity structure is through their behaviours at infinity.

Given that the solutions of first-order rational difference equations admit no essential branching, yet solutions of most first-order rational differential equations do show branching about movable singularities, it is natural to enquire into the process whereby branching arises in the process of taking a continuous limit in which a difference equation becomes a differential equation. To this end we consider the difference equation

$$
y(z+1)-y(z)=h F(y(z))
$$

where $h$ is a small parameter and $F$ is a rational function of $y(z)$. In order to take the (standard) continuum limit we perform the change of variables

$$
y(z)=u(x) \quad x=h z
$$

which gives

$$
\frac{u(x+h)-u(x)}{h}=F(u(x)) .
$$

We take the limit $h \rightarrow 0$ such that $x$ is fixed to obtain

$$
\frac{\mathrm{d} u}{\mathrm{~d} x}=F(u(x))
$$

Recall that solutions of equation (16) are single-valued (modulo the periodic function) while, for generic choices of $F$ (for example, $F(u)=u^{3}$ ), the general solution of equation (17) is branched. Note that since $x=h z$ is held fixed as $h \rightarrow 0$, then $z \rightarrow \infty$. Hence the behaviour of solutions of equation (17) for finite values of $x$ is related to the behaviour of solutions of equation (15) at $z=\infty$.

\section{Nevanlinna theory}

The relationship between the value distribution of meromorphic functions and their behaviour at infinity is the principal subject of Nevanlinna theory. In this section we describe some of the key quantities of Nevanlinna theory; namely the characteristic, order and type of a meromorphic function. We consider differential and difference equations whose meromorphic solutions are of finite order and type.

The Nevanlinna characteristic

$$
T(r, f)=N(r, f)+m(r, f)
$$

of a meromorphic function $f(z)$ is the sum of the counting function $N(r, f)$ which is a measure of the number of poles within $|z|<r$ and the proximity function $m(r, f)$ which has contributions from the segments of $|z|=r$ where $|f(z)|$ is large. Explicitly, the counting function is defined by

$$
N(r, f)=\int_{0}^{r} \frac{n(t, f)-n(0, f)}{t} \mathrm{~d} t+n(0, f) \log r
$$


where $n(r, f)$ is the number of poles (counting multiplicities) of $f$ in $|z| \leqslant r$. The proximity function is defined by

$$
m(r, f)=\frac{1}{2 \pi} \int_{0}^{2 \pi} \log ^{+}\left|f\left(r \mathrm{e}^{\mathrm{i} \theta}\right)\right| \mathrm{d} \theta
$$

where

$$
\log ^{+} x=\max (0, \log x) .
$$

The order of a meromorphic function $f$ is defined by

$$
\sigma(f)=\limsup _{r \rightarrow \infty} \frac{\log T(r, f)}{\log r} .
$$

If $f$ is entire, this agrees with the definition

$$
\sigma(f)=\limsup _{r \rightarrow \infty} \frac{\log \log M(r, f)}{\log r}
$$

where

$$
M(r, f)=\max _{|z|=r}|f(z)| .
$$

Since the order is a natural measure of the growth of a function at infinity, we consider differential and difference equations whose meromorphic solutions are of finite order. In this case the type of $f$ is defined to be

$$
\tau(f)=\limsup _{r \rightarrow \infty} r^{-\sigma} T(r, f) .
$$

It is a classical result due to Malmquist (see, for example, Hille [21]) that any first-order differential equation of the form

$$
\frac{\mathrm{d} y}{\mathrm{~d} z}=P(z, y)
$$

where $P$ is polynomial in $y(z)$ and analytic in $z$, that admits non-rational meromorphic solutions is a special case of the Riccati equation:

$$
\frac{\mathrm{d} y}{\mathrm{~d} z}=a_{0}(z)+a_{1}(z) y+a_{2}(z) y^{2} .
$$

All meromorphic solutions of the Riccati equation (where the coefficients $a_{j}$ are rational functions of $z$ ) are of finite order [39]. The Weierstrass and Jacobi elliptic functions are of order two and satisfy first-order second-degree equations of the form

$$
\left(\frac{\mathrm{d} y}{\mathrm{~d} z}\right)^{2}=P(y)
$$

where $P$ is a polynomial of degree at most four (see [21]). Moving on to second-order differential equations, we note that, of the six Painlevé equations $P_{\mathrm{I}}-P_{\mathrm{VI}}$, only $P_{\mathrm{I}}, P_{\mathrm{II}}$ and $P_{\text {IV }}$ have meromorphic general solutions. The order of the non-rational solutions of these equations are also finite (being $\frac{5}{2}, 3$ and 4 , respectively) [9,21]. We note that, as outlined above, Nevanlinna theory is not directly applicable to equations such as the $P_{\mathrm{VI}}$ because its general solution is branched about fixed singularities. 
Next let us consider difference equations. For first-order difference equations, Yanagihara [40] has shown that if the equation

$$
y(z+1)=\frac{a_{0}(z)+a_{1}(z) y+\cdots+a_{p}(z) y^{p}}{b_{0}(z)+b_{1}(z) y+\cdots+b_{q}(z) y^{q}}
$$

where $a_{i}$ and $b_{i}$ are polynomials, admits a non-rational meromorphic solution of finite order, then $\max (p, q)=1$ (i.e. equation (20) is the integrable difference Riccati equation). Note that this result implies that all non-rational meromorphic solutions of the logistic equation (12) are of infinite order for $\mu \neq 0$. For example, if $\mu=4$, the general solution of equation (12) is

$$
y(z)=\frac{1}{2}\left\{1-\cosh \left(2^{z} \pi(z)\right)\right\}
$$

where $\pi$ is an arbitrary periodic function. So for $\mu=4$, the logistic equation (12) is integrable in the sense that it is solvable but its solution grows very rapidly and is badly behaved numerically.

In the appendix we prove the following.

Theorem 3. If the second-order difference equation

$$
y(z+1)+y(z-1)=R(z, y(z)):=\frac{a_{0}(z)+a_{1}(z) y+\cdots+a_{p}(z) y^{p}}{b_{0}(z)+b_{1}(z) y+\cdots+b_{q}(z) y^{q}}
$$

where $a_{i}$ and $b_{i}$ are polynomials, admits a non-rational meromorphic solution of finite order, then $\max (p, q)=2$.

Note that this class of equations includes all the discretizations of $P_{\mathrm{I}}$ and $P_{\mathrm{II}}$ of the form (21) that are known to have associated linear problems. This class also contains equations solvable in terms of elliptic functions $[18,22,34]$. For example, from the addition formulae for Jacobi elliptic functions it follows that the equation

$$
y(z+1)+y(z-1)=\frac{2 y(z)}{1-y^{2}(z)}
$$

has the general solution

$$
y(z)=\pi_{1}(z) \operatorname{cn}\left[\Omega\left(z-\pi_{2}(z)\right), \kappa(z)\right]
$$

where $\pi_{1}$ and $\pi_{2}$ are arbitrary periodic functions, $\operatorname{cn}(\Omega)=\operatorname{dn}^{2}(\Omega)$, and the modulus of the elliptic function is $\kappa(z)=\mathrm{i} \pi_{1}(z) \operatorname{sn}(\Omega) / \operatorname{dn}(\Omega)$. For suitable $\pi_{1}$ and $\pi_{2}$ (e.g. constant), the general solution (23) is of finite order.

We remark that theorem 3 does not give a complete classification of second-order rational equations of the form (21) with respect to the order of solutions. In particular, we prove the following theorem in the appendix.

Theorem 4. Any entire non-polynomial solution of the equation

$$
y(z+1)+y(z-1)=a(z)+b(z) y(z)+c y^{2}(z)
$$

where $a$ and $b$ are polynomials and $c \neq 0$ is a constant, is of infinite order.

The proof of theorem 3 readily extends to a proof of the following.

Theorem 5. If the second-order difference equation

$$
y(z+1) y(z-1)=R(z, y(z)):=\frac{a_{0}(z)+a_{1}(z) y+\cdots+a_{p}(z) y^{p}}{b_{0}(z)+b_{1}(z) y+\cdots+b_{q}(z) y^{q}}
$$

where the $a_{i}, b_{i}$ are polynomials, admits a non-rational meromorphic solution of finite order, then $\max (p, q)=2$. 
The class of equations of the form (25) includes integrable difference versions of the third and fourth Painlevé equations [35]. If $R(z, y(z))=y^{2}(z)$ then equation (25) has the general solution $y(z)=\pi_{1}(z)\left[\pi_{2}(z)\right]^{z}$, where $\pi_{1}$ and $\pi_{2}$ are periodic. Hence, for $\pi_{1}$ and $\pi_{2}$ constant, $y$ has finite order. Note that theorems 3 and 5 do not single out only those equations that one would consider to be of Painlevé type.

Throughout this section we have only considered equations with polynomial or rational dependence on the independent variable. In general, the rate of growth of a meromorphic solution of differential and difference equations depends on the rate of growth of coefficients in the equation.

\section{Series expansions}

In this section we consider a particular perturbation of equation (22), namely

$$
y(z+1)+y(z-1)=\frac{(2+\epsilon f(z)) y(z)}{1-y^{2}(z)}
$$

where $\epsilon$ is a small parameter and $f$ is an analytic function. The techniques developed here can be generalized to analyse equation $(21)$ with $\max (p, q)=2$. It is sufficient for our purposes to consider this example. We will develop a series expansion for solutions of equation (26). In general, this expansion will be a Laurent series in $z-z_{0}$ and the digamma function $\psi\left(z-z_{0}\right)=\mathrm{d} \log \Gamma\left(z-z_{0}\right) / \mathrm{d} z$. In these expansions $\psi$ plays a role analogous to the role played by log in the expansions of solutions of differential equations. We will see that demanding that these expansions have no $\psi$ dependence leads to the known integrable cases of equation (26).

We begin by substituting the expansion

$$
y(z)=y_{0}(z)+\epsilon y_{1}(z)+\epsilon^{2} y_{2}(z)+\cdots+\epsilon^{n} y_{n}(z)+\cdots
$$

into equation (26). Equating the terms independent of $\epsilon$ shows that $y_{0}$ satisfies equation (22). The terms proportional to $\epsilon$ gives

$$
y_{1}(z+1)-2 \frac{\left(1+y_{0}^{2}(z)\right)}{\left(1-y_{0}^{2}(z)\right)^{2}} y_{1}(z)+y_{1}(z-1)=\frac{y_{0}(z) f(z)}{1-y_{0}^{2}(z)} .
$$

Differentiating equation (22) with respect to $z$ shows that $\mathrm{d} y_{0} / \mathrm{d} z$ solves the homogeneous part of equation (28). Following the method of variation of parameters, we substitute

$$
y_{1}(z)=u(z) y_{0}^{\prime}(z)
$$

into equation (28), which yields

$$
u(z)-u(z-1)=\frac{v(z)}{y_{0}^{\prime}(z) y_{0}^{\prime}(z-1)}
$$

where $v$ satisfies

$$
v(z+1)-v(z)=\frac{y_{0}(z) y_{0}^{\prime}(z) f(z)}{1-y_{0}^{2}(z)} .
$$

To illustrate our method in a concrete way, we choose the simplest non-trivial solution of equation (22),

$$
y_{0}(z)=\frac{1}{\xi} \quad \text { where } \quad \xi=z-z_{0}
$$


and $z_{0}$ is arbitrary. We will later drop this restriction and illustrate a method that uses properties of the general solution (23) of equation (22).

Substituting (32) into equation (31) gives

$$
\begin{aligned}
v(z+1)-v(z) & =\frac{f\left(\xi+z_{0}\right)}{(\xi-1) \xi(\xi+1)} \\
& =A(\xi)+\frac{1}{2}\left\{\frac{f\left(z_{0}+1\right)}{\xi-1}-\frac{2 f\left(z_{0}\right)}{\xi}+\frac{f\left(z_{0}-1\right)}{\xi+1}\right\}
\end{aligned}
$$

where $A$ is analytic. Equation (33) gives

$v(z)=Q(\xi)+\frac{1}{2}\left\{f\left(z_{0}+1\right) \psi(\xi-1)-2 f\left(z_{0}\right) \psi(\xi)+f\left(z_{0}-1\right) \psi(\xi+1)\right\}+\pi_{1}(z)$.

Hence, using the fact that the digamma function, $\psi$, satisfies

$$
\psi(\xi+1)-\psi(\xi)=\frac{1}{\xi}
$$

we have

$$
v(z)=B(\xi)+\frac{f\left(z_{0}-1\right)}{2 \xi}-\frac{f\left(z_{0}+1\right)}{2(\xi-1)}+c \psi(\xi)+\pi_{1}(\xi)
$$

where $\pi_{1}$ is an arbitrary periodic function, $B$ is analytic and

$$
c\left(z_{0}\right)=\frac{1}{2}\left\{f\left(z_{0}-1\right)-2 f\left(z_{0}\right)+f\left(z_{0}+1\right)\right\} .
$$

Note that if $c\left(z_{0}\right) \neq 0$ then from equations (29)-(34), $y_{1}$ will contain an explicit (linear) dependence on $\psi$. In fact, if $c\left(z_{0}\right) \neq 0$ then the expansion (27) for $y$ will contain infinitely many $\psi$-terms. If $c\left(z_{0}\right)=0$ for all $z_{0}$ then $f(z)=a z+b$, where $a$ and $b$ are periodic, which we choose to be constant, and equation (26) becomes

$$
y(z+1)+y(z-1)=\frac{(2+\epsilon(a+b z)) y(z)}{1-y^{2}(z)} .
$$

This is a known integrable difference equation which, for $b \neq 0$, has a continuum limit to the second Painlevé equation (2) with $\alpha=0$.

Next we discuss how to derive this result by perturbing off the general elliptic function solution (23) where for convenience we take $\pi_{1}$ and $\pi_{2}$ to be constant. We begin by locating the singular points of the right-hand side of equation (31). Since $y_{0}$ is a (scaled) Jacobi elliptic function, it takes on every value (including $\infty$ ) exactly once in each period parallelogram $\mathrm{P}$. There are at most three points $z_{ \pm}$and $z_{0}$ in $\mathrm{P}$ where the right-hand side of equation (31) can be singular. These points are defined by $y_{0}\left(z_{ \pm}\right)= \pm 1$ and $y_{0}\left(z_{0}\right)=\infty$ ( $y$ has a simple pole at $z_{0}$ ). On choosing initial conditions such that $\mathrm{P}$ is sufficiently large and balancing the poles on both sides of equation (31) we find that either $z_{ \pm}=z_{0} \pm 1$ or $z_{ \pm}=z_{0} \mp 1$. Without loss of generality we assume $z_{ \pm}=z_{0} \pm 1$ and hence $y$ has the expansions

$$
y_{0}(z)= \begin{cases}\frac{c_{0}}{z-z_{0}}+\mathrm{O}(1) & z \sim z_{0} \\ \pm 1+c_{ \pm}\left(z-\left[z_{0} \pm 1\right]\right)+\mathrm{O}\left(\left(z-\left[z_{0} \pm 1\right]\right)^{2}\right) & z \sim z_{0} \pm 1\end{cases}
$$

for some constants $c_{0}$ and $c_{ \pm}$. These expansions allow us to calculate residues of the poles of the right-hand side of equation (31) at $z_{0}$ and $z_{0} \pm 1$. Hence equation (31) has the form (33) where now $A$ is analytic throughout $\mathrm{P}$. The argument then proceeds as above. 
In this section we have described a method for difference equations which is analogous to that used by Painlevé in the context of branching of solutions of differential equations (see, e.g. Ince). The $\psi$ function plays the role of $\log$ in Painlevé's analysis. We note that $\psi$ is a meromorphic function of finite order but infinite type. Difference equations whose solutions are of finite order and type (up to the arbitrary periodic functions) are natural candidates to be further investigated regarding their integrability.

\section{Other approaches}

In this section we discuss two properties and their associated tests that have been introduced recently. These are the singularity confinement property and the discrete Painlevé property.

\subsection{The singularity confinement property}

Grammaticos and Ramani et al $[18,19,35]$ have proposed a test for the integrability of discrete equations that involves examining solutions (lists of iterates) that pass arbitrarily close to singularities of the equation. The basic idea is that for a generic (i.e. non-integrable) rational equation, if initial conditions are chosen so that the next iterate becomes infinite, then all future iterates will be infinite (such iterations of infinity are defined perturbatively—see the examples below). In this case the singularity is said to be not confined. Grammaticos and Ramani et al claim that the integrability of discrete equations is associated with the preservation of information contained within the initial conditions after the solution has passed through a singularity.

The singularity confinement property has been used by several authors and has identified many discrete equations which possess many of the properties that continuous integrable equations have, for example, the (so-called) discrete Painlevé equations. Indeed, the test was used to identify the first known integrable discretizations of the third, fourth and fifth Painlevé equations [35]. Hence this test has been successful in isolating interesting and important equations.

We remark, however, that recently Hietarinta and Viallet [20] have shown that the equation

$$
u_{n+1}+u_{n-1}=u_{n}+\frac{a}{u_{n}^{2}}
$$

possesses the singularity confinement property and yet it is (numerically) chaotic for $a \neq 0$. On the other hand, from theorem 3 we see that for $a \neq 0$, non-rational meromorphic solutions of equation (35) have infinite order. From our point of view, such equations should not be included within the class of Painlevé-type difference equations (the infinite-order growth of their solutions indicates that they are too 'ill-posed' in the complex plane). The singularity confinement test also contains a number of restrictions: for example, the maps must be strictly rational and there must be no 'proliferation of pre-images' (see [19]). In particular, the test cannot be used to analyse the logistic equation (12). On the other hand, the discussion here includes all equations of the form (10) as a special case of equation (20).

Consider the reversible, rational, second-order discrete equation

$$
y_{n+1}=y_{n}+\frac{y_{n}-y_{n-1}}{1+\left(y_{n}-y_{n-1}\right)} \text {. }
$$

This equation becomes singular when $1+y_{n}-y_{n-1}=0$. In order to explore this singularity we consider the initial conditions

$$
y_{0}=k \quad y_{1}=k-1+\epsilon
$$


where $k$ is an arbitrary parameter. Following the ideas of Grammaticos and co-workers $[18,36]$, we calculate the next few iterates: $y_{2}=-\epsilon^{-1}+k+\mathrm{O}(\epsilon), y_{3}=-\epsilon^{-1}+(k+1)+\mathrm{O}(\epsilon)$, $y_{4}=-\epsilon^{-1}+\left(k+\frac{3}{2}\right)+\mathrm{O}(\epsilon)$. It appears that the singularity is not confined in the sense of Grammaticos, Ramani et al. We will now show that this is indeed true, despite the fact that equation (36) has a simple solution.

Let

$$
w_{n}=\left(y_{n}-y_{n-1}\right)^{-1}
$$

Equation (36) becomes

$$
\Delta w_{n} \equiv w_{n+1}-w_{n}=1
$$

which, in turn, sums to give $w_{n}=n-n_{0}$, where $n_{0}$ is an arbitrary constant. Hence

$$
y_{n+1}-y_{n}=\frac{1}{n-n_{0}}
$$

which sums to give

$$
y_{n}=y_{0}+\sum_{m=0}^{n-1} \frac{1}{m-n_{0}} .
$$

We see that equation (36) is integrable and (from equation (37)) $y_{n}=-\epsilon^{-1}+\mathrm{O}(1)$, for $n \geqslant 2$ (so the singularity is not confined).

On the other hand, if we consider equation (36) as a difference equation, the solution of this equation is $(n \mapsto z)$

$$
y(z)=\pi_{1}(z)+\psi\left(z-\pi_{2}(z)\right)
$$

where $\pi_{1}$ and $\pi_{2}$ are arbitrary periodic functions and $\psi(z)=\mathrm{d} \log \Gamma(z) / \mathrm{d} z$ (digamma function). This example and others suggest the following. For difference equations with solutions of finite order, the singularity confinement test can be interpreted as a test for the appearance of $\psi$ in expansions of solutions of difference equations. We also note that in terms of the variable $z, y(z)$ is meromorphic for all $z$ modulo $\pi_{1}$ and $\pi_{2}$.

A (natural) continuum limit of equation (36) is

$$
y^{\prime \prime}+\left(y^{\prime}\right)^{2}=0
$$

which has the general solution

$$
y(z)=z_{1}+\log \left(z-z_{2}\right)
$$

where $z_{1}$ and $z_{2}$ are constants. Although equation (39) does not possess the Painlevé property, we still consider it to be integrable as the single log can be transformed away. Similarly, we consider equation (36) to be integrable because its general solution contains only one $\psi$ function (digamma function). However, many of the equations related to Painlevé equations can be considered perturbations of autonomous equations which have elliptic functions as solutions. In this case, if an expansion near a pole of the elliptic function gives rise to a series involving digamma functions arises, then there would be contributions from the infinity of poles of the elliptic function which we take to be an indication of non-integrability. This is a difference analogue of the psi-series which arise in the singularity analysis of differential equations. 


\subsection{The discrete Painlevé property}

Conte and Musette [12] define their discrete Painlevé property as follows. A difference equation,

$$
F\left(x, h,\left\{u(x+k h), k-k_{0}=0, \ldots, N\right\}\right)=0
$$

is said to possess the discrete Painlevé property if and only if there exists a neighbourhood of $h=0$ within which the general solution $x \mapsto u(x, h)$ has no movable critical singularities as a function of $x$. Thus this definition demands that the difference equation has a parameter $h$ which allows us to obtain a meaningful continuum limit as $h$ tends to zero. Hence we are restricted to a non-generic subclass of difference equations. In general, a difference equation will not have a parameter or a continuum limit. In the broad sense, the integrability of a difference equation should not depend on the existence of an arbitrary parameter in the equation. This definition also presents us with other difficulties if we are to associate the discrete Painlevé property with integrability.

Under this definition the trivial difference equation

$$
y(z+h)-y(z)=0
$$

fails to possess the discrete Painlevé property since its general solution is an arbitrary periodic function. For example,

$$
y(z)=\log \left(\cos \left(\frac{2 \pi z}{h}\right)\right)
$$

is a solution with logarithmic branching for arbitrarily small $h \neq 0$. This kind of difficulty can be removed by adding a caveat to the definition to the effect that we are only concerned with essential branching (branching due to sources other than the periodic functions). With this in mind consider the difference equation

$$
u(x+h)-u(x)+h u^{2}(x)=0 .
$$

Under the transformation $z=x / h, y(z)=h u(x)$, equation (41) becomes equation (13). Thus equation (41) admits the family of solutions

$$
u(x)=\frac{w(h x+\pi(h x))}{h}
$$

where $\pi$ is an arbitrary periodic function and $w$ is an entire function (cf equation (14) and [37]). Also, in the limit $h \rightarrow 0$, equation (41) becomes $u^{\prime}+u^{2}=0$ which has only rational solutions. So ignoring singularities due to the periodic function $\pi$ (by, for example, restricting $\pi$ to be entire), we see that $u$ is meromorphic for all $h$ and hence equation (41) possesses the discrete Painlevé property. Equation (41), however, is known to be non-integrable. From our point of view, equation (41) should be excluded from our analysis because its meromorphic solutions are of infinite order.

This test has, nonetheless proved to be successful and yields some information about the asymptotic structure of the solution at infinity. Recall from section 4 that if we take $x=h z$ to be held fixed as we let $h$ become arbitrarily small, then an expansion in $h$ would be an expansion in $1 / z$ in terms of the original, unscaled equation (15). These expansions are limits of the expansions described above in section 4 . 


\section{Summary and conclusion}

In this paper we have explored the singularity structure of solutions of certain classes of difference equations in the complex domain.

We note that for a large class of difference equations the singularity structure of the solutions is meromorphic in the complex domain, apart from singularities that may arise due to the arbitrary periodic functions. This leads us to conclude that the integrability of many difference equations is encoded in the nature of the singularity at infinity in the same way that the integrability of many differential equations is encoded in the movable singularities in the finite plane. Much research remains to be done to determine exactly what type of singularities at infinity are acceptable.

Our analysis has led us to consider a special class of equations whose solutions (up to the arbitrary periodic functions) are well behaved in the complex plane. In particular, the equations that we consider to be of Painlevé type have solutions that are:

(a) finite order in the sense of Nevanlinna, and

(b) have no digamma functions (i.e. $\psi$ 's) in their series expansions as outlined in section 4 . The digamma functions play an analogous role to the logs in psi-series (Painlevé) expansions of differential equations.

We have only considered difference analogues of logarithmic singularities in the series method described above. Meromorphic functions such as $\Gamma\left(z+\frac{1}{2}\right) / \Gamma(z)$, which plays the role of $\sqrt{z}$ for difference equations, will be considered elsewhere.

\section{Acknowledgment}

This work was partially supported by National Science Foundation grant no DMS-9703850.

\section{Appendix. Order of solutions of second-order rational difference equations}

In this appendix we provide a proof of theorem 3 based on arguments used by Yanagihara [40] for first-order equations. We begin by proving the following lemma.

Lemma 1. Given $\epsilon>0$ and a meromorphic function $y$, the Nevanlinna characteristic function $T$ satisfies

$$
T(r, y(z \pm 1)) \leqslant(1+\epsilon) T(r+1, y(z))+\kappa
$$

for all $r \geqslant 1 / \epsilon$, for some constant $\kappa$.

Proof. We will use the following identity which follows from Cartan's identical relation (see, for example, [42]):

$$
\frac{\mathrm{d} T(r, f(z))}{\mathrm{d} \log r}=\frac{1}{2 \pi} \int_{0}^{2 \pi} n\left(r, \mathrm{e}^{\mathrm{i} \theta}, f(z)\right) \mathrm{d} \theta
$$

where $n\left(r, \mathrm{e}^{\mathrm{i} \theta}, f(z)\right)$ is the number of solutions of

$$
f(z)=\mathrm{e}^{\mathrm{i} \theta}
$$

(counting multiplicities) inside $|z| \leqslant r$.

It follows from the definition of $n$ that $n\left(r+1, \mathrm{e}^{\mathrm{i} \theta}, y(z)\right)$ is the number of solutions of equation (A2) in $R:=\{z:|z| \leqslant r+1\}$ and $n\left(r, \mathrm{e}^{\mathrm{i} \theta}, y(z \pm 1)\right)$ is the number of solutions of 
$y(z \pm 1)=\mathrm{e}^{\mathrm{i} \theta}$ inside $|z| \leqslant r$ or, equivalently, the number of solutions of equation (A2) in $R_{ \pm}:=\{z:|z \mp 1| \leqslant r\}$. Using the fact that $R_{+}$and $R_{-}$are subsets of $R$, we conclude that $n(r, \mathrm{i} \theta, y(z \pm 1)) \leqslant n\left(r+1, \mathrm{e}^{\mathrm{i} \theta}, y(z)\right)$ for all $\theta$ and using the identity (A1) we find

$$
\frac{\mathrm{d} T(r, y(z \pm 1))}{\mathrm{d} \log r} \leqslant \frac{\mathrm{d} T(r+1, y(z))}{\mathrm{d} \log (r+1)} \leqslant\left(1+\frac{1}{r}\right) \frac{\mathrm{d} T(r, y(z))}{\mathrm{d} \log r} .
$$

Integrating this equation for $r \geqslant 1 / \epsilon$ establishes the lemma.

Proof of theorem 3. Equating the Nevanlinna characteristics of both sides of equation (21), we find

$$
T(r, y(z+1)+y(z-1))=T(r, R(z, y(z))=m T(r, y(z))+\mathrm{O}(\log r)
$$

where $m=\max (p, q)$ and the second equality in equation (A3) follows from a theorem of Valiron [38]. Using lemma 1, together with the fact that the left-hand side of equation (A3) is bounded above by $T(r, y(z+1))+T(r, y(z-1))+\log 2$ (see, for example, [21]) gives

$$
T(r+1, y(z)) \geqslant k T(r, y(z))+g(r)
$$

where $k=m / 2(1+\epsilon)$ and $|g(r)|<K \log r$ for some $K$ and all $r$ greater than some $r_{0}$. Hence for $r>r_{0}$,

$$
T(r+j, y(z)) \geqslant k^{j} T(r, y(z))+E_{j}(r)
$$

where

$$
\begin{aligned}
\left|E_{j}(r)\right| & =\left|k^{j-1} g(r)+k^{j-2} g(r+1)+\cdots+g(r+j-1)\right| \\
& \leqslant K k^{j-1} \sum_{i=0}^{j-1} \frac{\log (r+i)}{k^{i}} \\
& \leqslant K k^{j-1} \sum_{i=0}^{\infty} \frac{\log (r+i)}{k^{i}} .
\end{aligned}
$$

Using $\log (r+i) \leqslant \log r \log i$, for $r$ and $i$ sufficiently large, we note that since $k>1$ the series converges and hence

$$
\left|E_{j}(r)\right| \leqslant C k^{j} \log r
$$

where $C$ is a positive constant.

A meromorphic function $f$ is rational if and only if $T(r, f)=\mathrm{O}(\log r)$. Since, by hypothesis, $y$ is non-rational we can choose $r_{0}$ sufficiently large such that for all $r>r_{0}$, we have $T(r, y(z))>2 C \log r$ (where we have used the fact that $T$ is an increasing function of $\log r$, cf equation (A1)). Hence equation (A5) implies

$$
T(r+j, y(z))>C k^{j} \log r .
$$

and so

$$
\frac{\log T(r+j, y(z))}{\log (r+j)}>\frac{j \log k+\log (C \log r)}{\log (r+j)} .
$$

So for $k>1, \sigma(y)=\infty($ cf equation (18)).

Proof of theorem 4. Without loss of generality we rescale equation (24) so that $c=3$. Let

$$
M(r):=\max _{|z|=r}|y(z)| .
$$


By considering the maximum of $|y(z \pm 1)|$ on $|z|=r$ we see that the maximum principle gives

$$
M(r+1) \geqslant \max _{|z|=r}|y(z \pm 1)| .
$$

Hence

$$
\begin{aligned}
2 M(r+1) & \geqslant \max _{|z|=r}|y(z+1)|+\max _{|z|=r}|y(z-1)| \\
& \geqslant \max _{|z|=r}|y(z+1)+y(z-1)| \\
& \geqslant \max _{|z|=r}\left|a(z)+b(z) y(z)+3 y^{2}(z)\right|
\end{aligned}
$$

where we have used equation (24). Since $a$ and $b$ are polynomials and $y$ is not, there exists an $r_{0}$ such that

$$
M^{2}(r)=\max _{|z|=r}|y(z)|^{2} \geqslant \max _{|z|=r}|a(z)+b(z) y(z)|
$$

for all $r>r_{0}$. Hence, using equation (A6), we find for sufficiently large $r$

$$
M(r+1) \geqslant M^{2}(r)
$$

and so

$$
M(r+i) \geqslant M^{2^{i}}(r)
$$

This gives

$$
\frac{\log \log M(r+i)}{\log (r+i)} \geqslant \frac{i \log 2+\log \log M(r)}{\log (r+i)}
$$

which, from equation (19), shows that $y$ has infinite order.

The proof of theorem 5 uses the identity $T\left(r, f_{1} f_{2}\right) \leqslant T\left(r, f_{1}\right)+T\left(r, f_{2}\right)$ (see, for example, [21]) but is otherwise identical to the proof just given for theorem 3.

\section{References}

[1] Ablowitz M J, Chakravarty S and Halburd R 1998 Darboux-Halphen systems and the singularity structure of its solutions Proc. 4th Int. Conf. on Mathematical and Numerical Aspects of Wave Propagation ed J A DeSanto (Philadelphia, PA. SIAM) pp 408-12

[2] Ablowitz M J, Chakravarty S and Halburd R 1998 On Painlevé and Darboux-Halphen type equations The Painlevé Property, One Century Later (CRM Series in Mathematical Physics) ed R Conte (Berlin: Springer)

[3] Ablowitz M J, Chakravarty S and Herbst B M 1995 Integrability, computability and applications Acta Appl. Math. 39 5-37

[4] Ablowitz M J and Clarkson P A 1991 Solitons, Nonlinear Evolution Equations and Inverse Scattering (London Mathematical Society Lecture Note Series vol 149) (Cambridge: Cambridge University Press)

[5] Ablowitz M J, Ramani A and Segur H 1978 Nonlinear evolution equations and ordinary differential equations of Painlevé type Lett. Nouvo Cimento 23 333-8

[6] Ablowitz M J, Ramani A and Segur H 1980 A connection between nonlinear evolution equations and ordinary differential equations of P-type. I J. Math. Phys. 21 715-21

Ablowitz M J, Ramani A and Segur H 1980 A connection between nonlinear evolution equations and ordinary differential equations of P-type. II J. Math. Phys. 21 1006-15

[7] Ablowitz M J and Segur H 1977 Exact linearization of a Painlevé transcendent Phys. Rev. Lett. 38 1103-6

[8] Birkhoff G D 1920 Surface transformations and their dynamical applications Acta Math. 43 1-119

[9] Boutroux P 1904 Sur quelques propriétés des fonctions entières Acta Math. 28 97-224

[10] Chakravarty S and Ablowitz M J 1996 Integrability, monodromy, evolving deformations and self-dual Bianchi IX systems Phys. Rev. Lett. 76 857-60

[11] Conte R and Mussette M 1996 A new method to test discrete Painlevé equations Phys. Lett. A 223 439-48 
[12] Conte R and Mussette M 1998 Rules of discretization for Painlevé equations Theory of Nonlinear Special Functions: the Painlevé Transcendents (Montreal, 13-17 May 1996) ed L Vinet and P Winternitz (Berlin: Springer)

[13] Devaney R L 1986 An Introduction to Chaotic Dynamical Systems (Menlo Park, CA: Benjamin-Cummings)

[14] Flaschka H and Newell A C 1980 Monodromy- and spectrum preserving deformations. i Commun. Math. Phys. 76 65-116

[15] Fokas A S, Grammaticos B and Ramani A 1993 From continuous to discrete Painlevé equations J. Math. Anal. Appl. $180342-60$

[16] Fuchs L 1905 Sur quelques équations différentielles linéares du second ordre C. R. Acad. Sci., Paris 141 555-8

[17] Gambier B 1910 Sur les équations différentielles du second ordre et du premier degré dont l'intégrale générale est à points critiques fixes Acta Math. 33 1-55

[18] Grammaticos B, Ramani A and Papageorgiou V 1991 Do integrable mappings have the Painlevé property? Phys. Rev. Lett. 67 1825-8

[19] Grammaticos B, Ramani A and Tamizhmani K M 1994 Nonproliferation of pre-images in integrable mappings J. Phys. A: Math. Gen. 27 559-66

[20] Hietarinta J and Viallet C 1998 Singularity confinement and chaos in discrete systems Phys. Rev. Lett. 81 325-8

[21] Hille E 1959-62 Analytic Function Theory vols 1, 2 (Boston, MA: Ginn)

[22] Hirota R 1978 Nonlinear partial difference equations iv. Bäcklund transformation for the discrete-time Toda lequation J. Phys. Soc. Japan 45 321-32

[23] Jimbo M and Miwa T 1981 Monodromy preserving deformation of linear ordinary differential equations with rational coefficients II Phys. D 2 407-48

[24] Joshi N, Burtonclay D and Halburd R G 1992 Nonlinear nonautonomous discrete dynamical systems from a general discrete isomonodromy problem Lett. Math. Phys. 26 123-31

[25] Kimura T 1971 On the iteration of analytic functions Funkcial. Ekvac. 14 197-238

[26] Kitaev A V and Fokas A S 1990 An isomonodromy approach to the theory of two-dimensional quantum gravity Russ. Math. Surv. 45 155-7

[27] Kruskal M D, Joshi N and Halburd R 1997 Analytic and asymptotic methods for nonlinear singularity analysis: a review and extensions of tests for the Painlevé property Integrability of Nonlinear Systems (Pondicherry, 1996) (Lecture Notes in Physics vol 495) ed B Grammaticos and K Tamizhmani (Berlin: Springer) pp 171-205

[28] May R M 1976 Simple mathematical models with very complicated dynamics Nature 261 459-67

[29] Nijhoff F W, Satsuma J, Kajiwara K, Grammaticos B and Ramani A 1996 A study of the alternative discrete Painlevé-II equation Inverse Problems 12 697-716

[30] Painlevé P 1900 Mémoire sur les équations différentielles dont l'integrale générale est uniforme Bull. Soc. Math. France 28 201-61

[31] Painlevé P 1902 Sur les équations différentielles du second ordre et d'ordre supérieur dont l'integrale générale est uniforme Acta Math. 25 1-85

[32] Papageorgiou V G, Nijhoff F W, Grammaticos B and Ramani A 1992 Isomonodromic deformation problems for discrete analogues of Painlevé equations Phys. Lett. A 164 57-64

[33] Periwal V and Shevitz D 1990 Unitary matrix models as exactly solvable string theories Phys. Rev. Lett. 64 1326-9

[34] Potts R B 1987 Weierstrass elliptic difference equations Bull. Aust. Math. Soc. 35 43-8

[35] Ramani A, Grammaticos B and Hietarinta J 1991 Discrete versions of the Painlevé equations Phys. Rev. Lett. 67 1829-32

[36] Ramani A, Grammaticos B and Tamizhmani K M 1993 Painlevé analysis and singularity confinement: the ultimate conjecture J. Phys. A: Math. Gen. 26 L53-8

[37] Shimomura S 1981 Entire solutions of a polynomial difference equation J. Fac. Sci. Univ. Tokyo Sect. IA Math. $28253-66$

[38] Valiron G 1931 Sur la derivee des fonctions algebroïdes Bull. Soc. Math. France 59 17-39

[39] Wittich H 1954 Einige Eigenschaften der Lösungen von $w=a(z)+b(z) w+c(z) w^{2}$ Arch. Math. 5 226-32

[40] Yanagihara N 1980 Meromorphic solutions of some difference equations Funkcial. Ekvac. 23 309-26

[41] Yanagihara N 1985 Meromorphic solutions of some difference equations of the nth order Arch. Ration. Mech. Anal. 91 169-92

[42] Zhang G-H 1993 Theory of Entire and Meromorphic Functions: Deficient and Asymptotic Values and Singular Directions (Translations of Mathematical Monographs vol 122) (Providence, RI: American Mathematical Society) 